\title{
Modified Extended Kalman Filtering for Tracking with Insufficient and Intermittent Observations
}

\author{
Pengpeng Chen, ${ }^{1}$ Honglu Ma, ${ }^{1}$ Shouwan Gao, ${ }^{1}$ and Yan Huang ${ }^{2}$ \\ ${ }^{1}$ School of Computer Science and Technology, China University of Mining and Technology, Xuzhou 221116, China \\ ${ }^{2}$ Transportation Bureau of Dinghai District, Zhoushan 316000, China
}

Correspondence should be addressed to Shouwan Gao; gaoshouwan@cumt.edu.cn

Received 19 March 2015; Revised 3 June 2015; Accepted 14 June 2015

Academic Editor: Lihui Wang

Copyright (C) 2015 Pengpeng Chen et al. This is an open access article distributed under the Creative Commons Attribution License, which permits unrestricted use, distribution, and reproduction in any medium, provided the original work is properly cited.

This paper is concerned with the Kalman filtering problem for tracking a single target on the fixed-topology wireless sensor networks (WSNs). Both the insufficient anchor coverage and the packet dropouts have been taken into consideration in the filter design. The resulting tracking system is modeled as a multichannel nonlinear system with multiplicative noise. Noting that the channels may be correlated with each other, we use a general matrix to express the multiplicative noise. Then, a modified extended Kalman filtering algorithm is presented based on the obtained model to achieve high tracking accuracy. In particular, we evaluate the effect of various parameters on the tracking performance through simulation studies.

\section{Introduction}

Recent advancements of micro sensors technology have boosted the development of wireless sensor networks (WSNs). As is well known, WSNs can perform a variety of tasks that range from environment monitoring [1] and military surveillance [2] to hospital healthcare [3] and traffic control [4]. Nowadays, target tracking is a crucial practical application of WSNs, such as tracking emergency rescue workers, tracking military targets, and tracking moving devices in transportation systems. Indeed, we benefit greatly from the availability of accurate tracking. However, the problem of accurate and reliable tracking is still one of the major challenges in WSNs to be fully addressed due to the finite system resources and environment constraints.

Prior work for tracking mobile target in WSNs can be roughly categorized into three types in the light of the mechanism adopted: the trilateration-based schemes [5], the modelbased methods like Kalman filter [6-8] and particle filtering [9-11], and techniques based on the additional hardware on the target [12]. It is noticed that all the tracking methods given in the aforementioned references are derived based on the assumption that the WSNs are reliable to get the satisfactory tracking results. The effect of packet dropouts is neglected due to the complicated analysis or modeling. In fact, packet dropouts are unavoidable in data transmission due to the unreliable characteristics of networks. Recently, some results are obtained on the tracking problem for WSNs with packet dropouts by using Kalman filter or its variations. Motivated by tracking applications of WSNs, paper [13] studies the problem of performing Kalman filtering with intermittent observations, where the measurements are assumed to be received in full or lost completely. Paper [14] extends the results in [13] to allow partial observation losses, where the observation processes can be sent in two packets which are lost separately. For the general case that observations are sent over more than two different wireless channels, the Kalman filter is designed in [15] where the time delay is considered simultaneously. As we can see, the problem of packet dropouts is discussed in the papers listed above. However, all these papers assume that the tracking systems are linear systems with multiplicative noise, where multiplicative noise is employed to describe the phenomenon of packet losses. In the practical tracking applications, the linear conditions are not always met; hence, the extended Kalman filter (EKF) suitable for nonlinear systems [16] is needed to account for the nonlinearity in the tracking systems. 
Besides the packet dropouts resulting in intermittent observations, insufficient anchor coverage is also a main issue which affects the tracking accuracy in WSNs. As is known, the target cannot always be detected by $m$ anchors simultaneously in practical application scenarios due to lack of costly anchors and environment constraints [17], where $m$ is the requisite number of the anchors covering each location point, typically, $m=3$ for most of the tracking schemes based on trilateration. Therefore, it is desirable to develop a filtering algorithm taking into account the above two constraints: packet dropouts and insufficient anchor coverage. To the best of authors' knowledge, this topic has not been fully investigated in the existing literature. This is the motivation of the present research.

In this paper, we design the modified extended Kalman filter (MEKF) for tracking systems suffering from both multiple packet dropouts and insufficient anchor coverage. The main contributions of this paper are as follows: (1) the phenomenon of packet dropouts and the condition of insufficient anchor coverage are modeled as two different Bernoulli random processes; thus it is reasonable to define the effective packet arrival indicator, indicating whether the desired measurements are received correctly or not, as the products of above two Bernoulli variables; (2) a general measurement model with multiplicative noise is proposed to describe the case of intermittent and insufficient observations; compared with some existing ones, the obtained model is more practical due to two facts: (a) the nonlinearity in the tracking systems is considered; (b) a matrix rather than a scalar quantity is employed to express the multiplicative noise since the matrix can describe the characteristics of different channels; (3) the modified extended Kalman filtering algorithm is proposed based on the obtained system model. Moreover, the effects of various parameters on the tracking performance are evaluated through simulation studies.

The paper is organized as follows. Section 2 describes the system scenario. The classical EKF is introduced in Section 3. In Section 4, the measurement model considering insufficient and intermittent observations is given, and the corresponding MEKF is designed. Numerical examples illustrating and evaluating the proposed filter are given in Section 5. Section 6 concludes the paper.

\section{System Description}

We consider the tracking problem in a distributed network system setting with fixed-topology anchors and filters/controllers. Even if it were simpler, the fixed-topology framework well addresses many relevant applications, for instance, environmental monitoring [18]. As shown in Figure 1, anchors and filters/controllers are located at different physical locations and thus communicate over wireless networks. Assume a system model underlying Kalman filtering where the nonobservable state of the moving target is denoted by $x_{k}$ and the observation vector $z_{k}$ consists of the noisy measurements. In the scenario of Figure 1, the components of $z_{k}$ are the measurements collected by the No. $k_{i}(i=1,2, \ldots, m)$ anchors which will be used to estimate

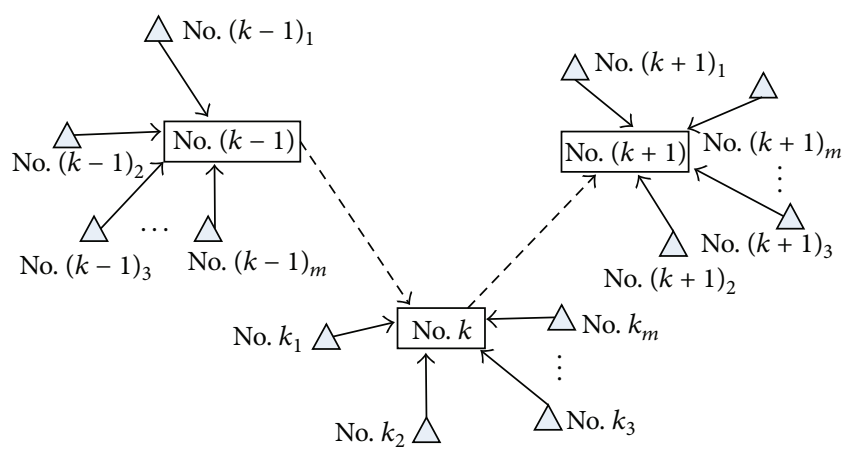

$\triangle$ Anchor

Filter/controller

Figure 1: Distributed network systems.

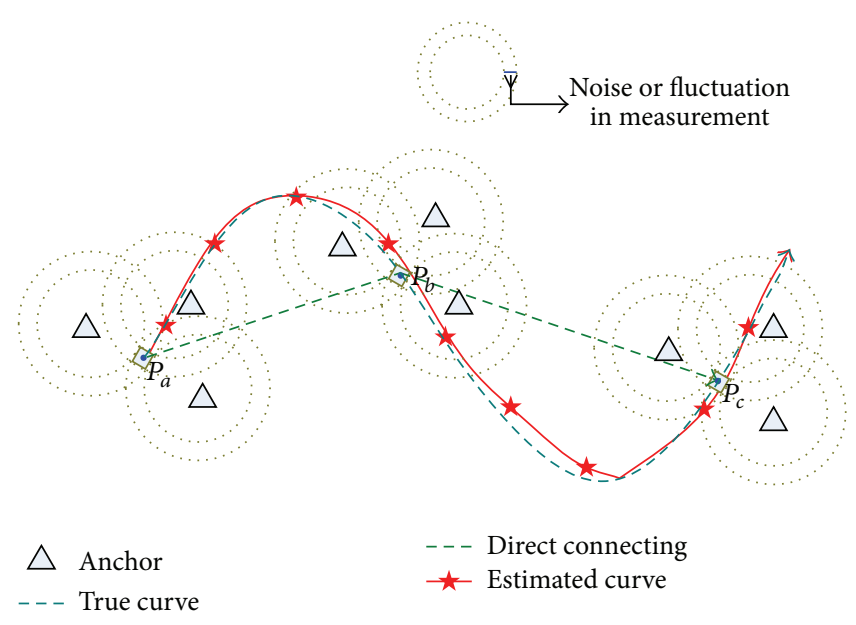

Figure 2: Comparison of tracking performance.

$x_{k}$. The No. $k$ filter in charge of estimating $x_{k}$ can directly exchange information with the filters handling the states $x_{k-1}$ and $x_{k+1}$. Due to the dynamic noise and environment constraints, the target cannot always be detected by $m$ anchors simultaneously. Besides, packet losses are inevitable because of collisions and transmission errors. Suffering from above two constraints, the target cannot always be localized based on trilateration. It should also be pointed out that since the measurements are noisy and fluctuate, the target can only be localized at possible location region rather than at a single point by trilateration, even if under three or more measurements. Figure 2 where $m=3$ depicts the estimated trace by directly connecting the centroid of possible locations (i.e., $P_{a}, P_{b}$, and $P_{c}$ ) under three measurements fails to provide a high tracking accuracy. Aiming at enhancing the tracking accuracy, we design the MEKF for the target tracking; and the corresponding estimation curve is shown as solid line with pentagram marker in Figure 2. 


\section{Overview of the Classical Extended Kalman Filtering Algorithm}

In this section, we introduce the classical extended Kalman filtering theory which assumes periodic measurement updates; that is, the target can be detected by $m$ anchors at any given time step $k$, and all the measurement packets can be received successfully.

3.1. The Moving Model for Mobile Target. The low dynamic scenario (pedestrian speeds) is considered throughout the paper; hence the position-velocity (PV) model is adopted to describe the two-dimensional motion of the target [19], which can be expressed by the following equation:

$$
x_{k}=F_{k} x_{k-1}+G_{k} \gamma_{k-1},
$$

where $x_{k}$ is the state vector and it is composed by the position coordinates and the velocity components; that is, $x_{k}=$ $\left[\begin{array}{llll}x_{k} & y_{k} & \dot{x}_{k} & \dot{y}_{k}\end{array}\right]^{T}$, in which $x_{k}$ and $y_{k}$ are the target's position coordinates in horizontal and vertical directions, respectively. Correspondingly, $\dot{x}_{k}$ and $\dot{y}_{k}$ are the speeds in horizontal and vertical directions, respectively. Assume that the target moves at near constant velocity between two adjacent estimation steps; the state transition matrix $F_{k}$ can be expressed by

$$
F_{k}=\left[\begin{array}{cccc}
1 & 0 & \Delta t_{k} & 0 \\
0 & 1 & 0 & \Delta t_{k} \\
0 & 0 & 1 & 0 \\
0 & 0 & 0 & 1
\end{array}\right],
$$

where $\Delta t_{k}=t_{k}-t_{k-1}$ is the interval between the current estimation time $t_{k}$ and the previous estimation time $t_{k-1}$.

As is well known, the moving target is often subject to different forces (e.g., frictions) that could temporally affect target's dynamics. Hence, the process noise which takes into account the perturbation on the system needs to be incorporated in the PV model. Without loss of generality, we model the process noise as the independent random acceleration $\gamma_{k}=\left[\begin{array}{ll}\ddot{x}_{k} & \ddot{y}_{k}\end{array}\right]^{T}$ with zero mean and covariance matrix $q_{k}$. From the above analysis, we can get that $G_{k}$ has the following form:

$$
G_{k}=\left[\begin{array}{cccc}
\frac{\Delta t_{k}^{2}}{2} & 0 & \Delta t_{k} & 0 \\
0 & \frac{\Delta t_{k}^{2}}{2} & 0 & \Delta t_{k}
\end{array}\right]^{T} .
$$

For the sake of simplicity, let us define

$$
w_{k-1}=G_{k} \gamma_{k-1} .
$$

Then, it follows from (4) that

$$
x_{k}=F_{k} x_{k-1}+w_{k-1} .
$$

It is easy to verify that $w_{k}$ is the independent noise with zero mean and covariance:

$$
Q_{k}=G_{k+1} q_{k} G_{k+1}^{T} .
$$

3.2. The Observation Model for WSNs. This subsection presents the position and distance observation models for the ideal case of Figure 1. At any given time step $k$, we assume that the target can be simultaneously detected by $m$ anchors which are located at known coordinates $\left(x_{\mathrm{anc}_{i, k},}, y_{\mathrm{anc}_{i, k}}\right)(i=$ $1,2, \ldots, m)$, respectively. If no observations are lost, $m$ measured distances $d_{i, k}(i=1,2, \ldots, m)$ between the target location $\left(x_{k}, y_{k}\right)$ and the locations of $m$ anchors can be used to estimate the target position by the following equation:

$$
d_{i, k}=\sqrt{\left(x_{k}-x_{\mathrm{anc}_{i, k}}\right)^{2}+\left(y_{k}-y_{\mathrm{anc}_{i, k}}\right)^{2}}+\widetilde{d}_{i, k}
$$

where $\widetilde{d}_{i, k}$ is the distance measurement error. Then, we rewrite (7) as

$$
z_{k}=h\left(x_{k}\right)+v_{k},
$$

where $z_{k}$ is the observation vector, and it is defined as $z_{k}=\left[\begin{array}{llll}d_{1, k} & d_{2, k} & \cdots & d_{m, k}\end{array}\right]^{T} . h$ is the observation function that relates the current state to the output. And $v_{k}=$ $\left[\begin{array}{llll}\tilde{d}_{1, k} & \tilde{d}_{2, k} & \cdots & \tilde{d}_{m, k}\end{array}\right]^{T}$ is the observation noise which is typically assumed as the independent white Gaussian noise with zero mean and covariance matrix $R_{k}$.

3.3. Extended Kalman Filter. The classical EKF design is described in this subsection for tracking the moving target. As described above, the tracking system can be formulated as the state equation (5) and the observation equation (8). Under the assumption that the random processes $\left\{w_{k}\right\}$ and $\left\{v_{k}\right\}$ for all $k$ and the initial state $x_{0}$ are mutually independent, the following equations can be calculated iteratively to track the target.

(1) State Prediction Step. Assume that state estimation $\widehat{x}_{k-1 \mid k-1}$ and the error covariance $P_{k-1 \mid k-1}$ are available at time step $(k-$ 1 ); the one-step state prediction $\hat{x}_{k \mid k-1}$ and the one-step error covariance prediction $P_{k \mid k-1}$ can be estimated as follows:

$$
\begin{aligned}
& \widehat{x}_{k \mid k-1}=F_{k} \widehat{x}_{k-1 \mid k-1}, \\
& P_{k \mid k-1}=F_{k} P_{k-1 \mid k-1} F_{k}^{T}+Q_{k-1} .
\end{aligned}
$$

(2) Measurement Update Step. When the new distance measurements are obtained, they can be used to update the state prediction. Correspondingly, the error covariance is also updated. The state estimation $\widehat{x}_{k \mid k}$ and the error covariance $P_{k \mid k}$ at time step $k$ can be calculated as

$$
\begin{aligned}
& \widehat{x}_{k \mid k}=\widehat{x}_{k \mid k-1}+K_{k} \widetilde{z}_{k}, \\
& P_{k \mid k}=\left(I-K_{k} H_{k}\right) P_{k \mid k-1},
\end{aligned}
$$

where $K_{k}$ and $\widetilde{z}_{k}$ are, respectively, the EKF gain and the innovation and they are defined as follows:

$$
\begin{aligned}
K_{k} & =P_{k \mid k-1} H_{k}^{T}\left(H_{k} P_{k \mid k-1} H_{k}^{T}+R_{k}\right)^{-1}, \\
\tilde{z}_{k} & =z_{k}-h\left(\widehat{x}_{k \mid k-1}\right) .
\end{aligned}
$$


It is obvious that the function $h$ in (8) is nonlinear, so its linear approximation $H_{k}$ is needed for the iterative calculation of the EKF. In the general case, $H_{k}$ can be chosen as the Jacobian matrix of the observation function evaluated around the state prediction $\widehat{x}_{k \mid k-1}$; that is,

$$
H_{k}=\frac{\partial h\left(x_{k}\right)}{\partial \widehat{x}_{k \mid k-1}}=\left[\begin{array}{cccc}
\frac{\partial d_{1, k}}{\partial \widehat{x}_{k \mid k-1}} & \frac{\partial d_{1, k}}{\partial \widehat{y}_{k \mid k-1}} & 0 & 0 \\
\frac{\partial d_{2, k}}{\partial \widehat{x}_{k \mid k-1}} & \frac{\partial d_{2, k}}{\partial \widehat{y}_{k \mid k-1}} & 0 & 0 \\
\vdots & \vdots & \vdots & \vdots \\
\frac{\partial d_{m, k}}{\partial \widehat{x}_{k \mid k-1}} & \frac{\partial d_{m, k}}{\partial \widehat{y}_{k \mid k-1}} & 0 & 0
\end{array}\right],
$$

where the partial derivatives are given below:

$$
\begin{gathered}
\frac{\partial d_{i, k}}{\partial \hat{x}_{k \mid k-1}}=\frac{\widehat{x}_{k \mid k-1}-x_{\mathrm{anc}_{i, k}}}{\sqrt{\left(\hat{x}_{k \mid k-1}-x_{\mathrm{anc}_{i, k}}\right)^{2}+\left(\widehat{y}_{k \mid k-1}-y_{\mathrm{anc}_{i, k}}\right)^{2}}}, \\
\frac{\partial d_{i, k}}{\partial \hat{y}_{k \mid k-1}}=\frac{\widehat{y}_{k \mid k-1}-y_{\mathrm{anc}_{i, k}}}{\sqrt{\left(\hat{x}_{k \mid k-1}-x_{\mathrm{anc}_{i, k}}\right)^{2}+\left(\hat{y}_{k \mid k-1}-y_{\mathrm{anc}_{i, k}}\right)^{2}}} .
\end{gathered}
$$

\section{Tracking with Insufficient and Intermittent Observations}

In this section, we modify the classical EKF to account for possible insufficient and intermittent observations. We first improve the observation equation to make it suitable for both the case of insufficient anchor coverage and the case of unreliable network connections. Then we design the MEKF based on the observation model obtained.

4.1. The Observation Model with Multiplicative Noise. Under the scenario of insufficient sensor coverage, the anchors transmit measurement signals if they can detect the target; otherwise they transmit empty packets with special flags (we will call them ineffective packets in this paper) to the filter. We use $\alpha_{i, k}$ to indicate whether the measurement packet sent by the No. $k_{i}$ anchor is ineffective packet at time step $k$. Moreover, we assume $\alpha_{i, k}(i=1,2, \ldots, m)$ is independent and identically distributed (i.i.d.) Bernoulli random variable with $E\left\{\alpha_{i, k}\right\}=\varepsilon_{i}$. Note that packet dropouts are inevitable due to transmission errors and collisions. We use the i.i.d. Bernoulli variables $\beta_{i, k}(i=1,2, \ldots, m)$ with means $b_{i}$ to represent whether the signal transmitted over the No. $k_{i}$ channel is received correctly. Denote the effective packet arrival indicator of the No. $k_{i}$ channel by $\mu_{i, k}$; obviously, $\mu_{i, k}=$ $\alpha_{i, k} \beta_{i, k}$.

Remark 1. The effective packet arrival indicator $\mu_{i, k}$ contains two important aspects: (1) describing the phenomenon of packet dropouts in the No. $k_{i}$ channel; (2) showing whether the No. $k_{i}$ anchor can detect the target or not. Obviously, the modeling and analysis method proposed is suitable for both the conditions of insufficient sensor coverage and the conditions of unreliable network connections.

Note that the channels may be correlated with each other; we use a general matrix $U_{k}=\left(u_{k}^{i j}\right)_{m \times m}$ to describe the multiplicative noise matrix of the tracking system; that is,

$$
z_{k}=U_{k} h\left(x_{k}\right)+v_{k}
$$

where the diagonal element of $U_{k}$ can be expressed by $u_{k}^{i i}=$ $\mu_{i, k}$ and the nondiagonal component $u_{k}^{i j}(i \neq j)$ indicates the correlation of the channels $i$ and $j$. Without loss of generality, the following assumptions are made on the multiplicative noise matrix $U_{k}$ throughout this subsection.

Assumption 2. $E\left\{U_{k}\right\}=M=\left(\psi^{i j}\right)_{m \times m}$, where $\psi^{i j}=E\left\{u_{k}^{i j}\right\}$.

Assumption 3. $E\left\{u_{k}^{i j} u_{t}^{r l}\right\}=\phi^{i j, r l} \delta_{k, t}$, where $\delta$ is the Kronecker Delta function.

Assumption 4. $U_{k}$ is independent of $w_{k}, v_{k}$, and $x_{0}$.

It is clear that Assumption 3 indicates that all channels of the multiplicative noise matrix are correlated with each other at the same time.

4.2. Modified Extended Kalman Filter. Consider the system given by (5) and (16). Since $h$ in (16) is a nonlinear function, we first expand the nonlinearities in $h$ about $\widehat{x}_{k \mid k-1}$ using the Taylor series expansion method [20]. Retaining only the first order terms and constant terms, we can approximate (16) as

$$
z_{k}=U_{k}\left(H_{k} x_{k}+f_{k}\right)+v_{k}
$$

where $H_{k}$ can be expressed by (13) and

$$
f_{k}=h\left(\widehat{x}_{k \mid k-1}\right)-H_{k} \widehat{x}_{k \mid k-1} .
$$

Suppose that the assumptions on $w_{k}, v_{k}$, and $x_{0}$ in Section 3 still hold. Before formulating the problem of the state estimation with insufficient and intermittent observations, we define the following innovation sequence $e_{k}$ associated with the measurement $z_{k}$ :

$$
e_{k}=z_{k}-\widehat{z}_{k \mid k-1}
$$

where the one-step measurement prediction $\widehat{z}_{k \mid k-1}$ can be described as

$$
\widehat{z}_{k \mid k-1}=U_{k} h\left(\widehat{x}_{k \mid k-1}\right) .
$$

Obviously, the state estimation $\widehat{x}_{k \mid k}$ of $x_{k}$ can be expressed as

$$
\widehat{x}_{k \mid k}=\widehat{x}_{k \mid k-1}+K_{k} e_{k},
$$

where the one-step state prediction $\widehat{x}_{k \mid k-1}$ is calculated by (9) and the filter gain $K_{k}$ is chosen to minimize the following function:

$$
E\left\{\left(x_{k}-\widehat{x}_{k \mid k}\right)\left(x_{k}-\widehat{x}_{k \mid k}\right)^{T}\right\} .
$$

The estimation problem is now formulated as follows: find the estimation $\widehat{x}_{k \mid k}$ as in (21), wherein the filter gain $K_{k}$ can minimize (22). 
Remark 5. Obviously, as shown in Figure 1, the multiplicative noise $U_{k}$ together with the observation $z_{k}$ is available for the No. $k$ filter at time step $k$, which is different from the iterative filter in [21], where the multiplicative noise cannot be obtained; hence the one-step measurement prediction $\widehat{z}_{k \mid k-1}$ is formulated as

$$
\widehat{z}_{k \mid k-1}=M h\left(\widehat{x}_{k \mid k-1}\right),
$$

where $M$ is the mean of $U_{k}$. It is not surprising that our filter performs better since $U_{k}$ is available in the one-step measurement prediction while the multiplicative noise in [21] is the unknown stochastic matrix for the filter; hence the expectation of $U_{k}$ is adopted for the iterative calculating.

Remark 6. The above estimation problem is also different from the suboptimal filtering problem studied in [22], where the measurement model is linear. Though it is simpler, the linear model has important limitations in many typical applications of sensor networks. For example, it cannot well address the problem of localization and tracking. Due to the practical requirement of tracking in WSNs, we adopt the nonlinear measurement model (16).

We are now in the position to present the filtering method for the multichannel nonlinear systems with multiplicative noise.

Theorem 7. For systems (5) and (16), the iterative modified extended Kalman filtering algorithm is given as below:

$$
\widehat{x}_{k \mid k}=\widehat{x}_{k \mid k-1}+K_{k}\left[z_{k}-U_{k} h\left(\widehat{x}_{k \mid k-1}\right)\right], \quad \widehat{x}_{0 \mid 0}=\xi_{0},
$$

where the one-step state prediction $\widehat{x}_{k \mid k-1}$ is formulated as in (9) and the gain matrix $K_{k}$ is calculated as

$$
K_{k}=P_{k \mid k-1} H_{k}^{T} M^{T}\left(S_{k}+R_{k}\right)^{-1},
$$

in which $S_{k}$ is defined as in (34) below. Moreover, the onestep error covariance prediction $P_{k \mid k-1}$ satisfies the recursive equation (10), and the error covariance matrix $P_{k \mid k}$ can be expressed by

$$
P_{k \mid k}=\left(I-K_{k} M H_{k}\right) P_{k \mid k-1}, \quad P_{0 \mid 0}=P_{0} .
$$

Proof. We firstly give the following definitions, which are useful for the development of our work:

$$
\begin{aligned}
\tilde{x}_{k \mid k-1} & =x_{k}-\widehat{x}_{k \mid k-1}, \\
\tilde{x}_{k \mid k} & =x_{k}-\widehat{x}_{k \mid k}, \\
P_{k \mid k-1} & =E\left\{\tilde{x}_{k \mid k-1} \tilde{x}_{k \mid k-1}^{T}\right\}, \\
P_{k \mid k} & =E\left\{\tilde{x}_{k \mid k} \tilde{x}_{k \mid k}^{T}\right\} .
\end{aligned}
$$

From (28) and (30), it is obvious that $P_{k \mid k}$ is equivalent to (22); hence our objective is now to find $K_{k}$ to minimize $P_{k \mid k}$.

Substituting (21) into (28) and noting (27), one can obtain

$$
\tilde{x}_{k \mid k}=\left(I-K_{k} U_{k} H_{k}\right) \tilde{x}_{k \mid k-1}-K_{k} v_{k},
$$

where $I$ is the identity matrix of appropriate dimensions.
From the aforementioned hypotheses on $U_{k}, w_{k}, v_{k}$, and $x_{0}$, it is easy to check that

$$
E\left\{\left(I-K_{k} U_{k} H_{k}\right) \tilde{x}_{k \mid k-1} v_{k}^{T}\right\}=0 .
$$

Thus, it follows from (31) that

$$
\begin{aligned}
P_{k \mid k}= & P_{k \mid k-1}+K_{k}\left(S_{k}+R_{k}\right) K_{k}^{T}-P_{k \mid k-1} H_{k}^{T} M^{T} K_{k}^{T} \\
& -K_{k} M H_{k} P_{k \mid k-1},
\end{aligned}
$$

where $S_{k}$ is defined as

$$
S_{k}=E\left\{U_{k} H_{k} \tilde{x}_{k \mid k-1} \tilde{x}_{k \mid k-1}^{T} H_{k}^{T} U_{k}^{T}\right\}=\left(s_{k}^{i j}\right)_{m \times m} .
$$

Letting

$$
G_{k}=H_{k} P_{k \mid k-1} H_{k}^{T}=\left(g_{k}^{i j}\right)_{m \times m},
$$

we obtain that the element $s_{k}^{i j}$ of the matrix $S_{k}$ in (34) satisfies the following equation:

$$
\begin{aligned}
& s_{k}^{i j} \\
& =E\left\{\sum_{l=1}^{m} \sum_{r=1}^{m} u_{k}^{i r} g_{k}^{r l} u_{k}^{j l}\right\} \\
& =\operatorname{Trace}\left[\left(\begin{array}{cccc}
\phi^{i 1, j 1} & \phi^{i 2, j 1} & \cdots & \phi^{i m, j 1} \\
\phi^{i 1, j 2} & \phi^{i 2, j 2} & \cdots & \phi^{i m, j 2} \\
\vdots & \vdots & \ddots & \vdots \\
\phi^{i 1, j m} & \phi^{i 2, j m} & \cdots & \phi^{i m, j m}
\end{array}\right)\left(H_{k} P_{k \mid k-1} H_{k}^{T}\right)\right] .
\end{aligned}
$$

In order to find $K_{k}$ to minimize (33), we rewrite (33) as

$$
\begin{aligned}
P_{k \mid k}= & P_{k \mid k-1}-P_{k \mid k-1} H_{k}^{T} M^{T}\left(S_{k}+R_{k}\right)^{-1} M H_{k} P_{k \mid k-1} \\
+ & {\left[K_{k}-P_{k \mid k-1} H_{k}^{T} M^{T}\left(S_{k}+R_{k}\right)^{-1}\right]\left(S_{k}+R_{k}\right) } \\
& \cdot\left[K_{k}-P_{k \mid k-1} H_{k}^{T} M^{T}\left(S_{k}+R_{k}\right)^{-1}\right]^{T} .
\end{aligned}
$$

It then follows from (37) that the minimizer can be found by choosing $K_{k}$ as in (25), and corresponding minimum error covariance $P_{k \mid k}$ can be expressed by (26), which concludes the proof.

4.3. A Special Case: Uncorrelated Communication Channels. As discussed above, the multiplicative noise matrix is assumed to be a general matrix, which corresponds to the practical case in which different communication channels (including the corresponding anchors) are correlated. When the channels are independent of each other, the multiplicative noise matrix can be described as a diagonal matrix which is the special form of the general matrix. Obviously, Theorem 7 can be directly applied for this case. However, $S_{k}$ in (34) can be actually expressed by a simple form due to the characteristics of the diagonal matrix.

Throughout this subsection, we assume that $U_{k}^{*}=$ $\operatorname{diag}\left\{\mu_{1, k}, \mu_{2, k}, \ldots, \mu_{m, k}\right\}$ is the multiplicative noise matrix and 
the effective packet arrival indicators $\mu_{i, k}(i=1,2, \ldots, m)$ are i.i.d. Bernoulli random variables with the mean and covariance as follows:

$$
\begin{aligned}
E\left\{\mu_{i, k}\right\} & =\lambda_{i}, \\
E\left\{\left(\mu_{i, k}-\lambda_{i}\right)\left(\mu_{j, t}-\lambda_{j}\right)\right\} & =\lambda_{i}\left(1-\lambda_{i}\right) \delta_{i, j} \delta_{k, t} .
\end{aligned}
$$

Hence, we obtain

$$
M^{*}=E\left\{U_{k}^{*}\right\}=\operatorname{diag}\left\{\lambda_{1}, \lambda_{2}, \ldots, \lambda_{m}\right\}
$$

Define

$$
S_{k}^{*}=E\left\{U_{k}^{*} H_{k} \tilde{x}_{k \mid k-1} \tilde{x}_{k \mid k-1}^{T} H_{k}^{T} U_{k}^{*}\right\}=\left(\theta_{k}^{i j}\right)_{m \times m},
$$

and note the characteristics of the diagonal matrix. Then, we have

$$
\theta_{k}^{i j}= \begin{cases}\lambda_{i} g_{k}^{i j} & i=j \\ \lambda_{i} \lambda_{j} g_{k}^{i j} & i \neq j\end{cases}
$$

where $g_{k}^{i j}$ is defined as in (35). It is noteworthy that (41) is much simpler than (36) which corresponds to the case of a general matrix.

Remark 8. Note that, as a special form, the diagonal matrix has all elements being zeros except for the diagonal elements. As a result, the filtering problem discussed in this subsection can be actually thought of as the particular case of the estimation studied in Section 4.2. It is important to stress that $M^{*}$ in (39) is symmetric matrix due to the diagonal form (while $M$ in (25) is not necessarily symmetric); thus (25) in Theorem 7 can be simply modified as

$$
K_{k}^{*}=P_{k \mid k-1} H_{k}^{T} M^{*}\left(S_{k}^{*}+R_{k}\right)^{-1}
$$

\section{Numerical Examples}

In this section, the results from the previous section are illustrated through simulation studies. Moreover, we evaluate the effect of various noise parameters on tracking performances for both the MEKF and the trilateration-based method.

5.1. Simulation Example for MEKF. Suppose the sample period $\Delta t_{k}$ is constant and the desired number of the observations is $m=3$. Consider the system (1) and (16), where $\gamma_{k}$ and $v_{k}$ are random noise with zero means and covariances $q_{k}=\operatorname{diag}\{1,1\}$ and $R_{k}=\operatorname{diag}\{0.1,0.1,0.1\}$, respectively. The mean of the multiplicative noise has the form of $M=\operatorname{diag}\{0.9,0.85,0.9\}$. We design the MEKF for the above system, and its tracking performance is shown in Figure 3, which shows that our filter is effective.

5.2. Performance Evaluation under Various Parameters. We evaluate the tracking accuracies of the MEKF and the trilateration-based method under different noise scenarios.

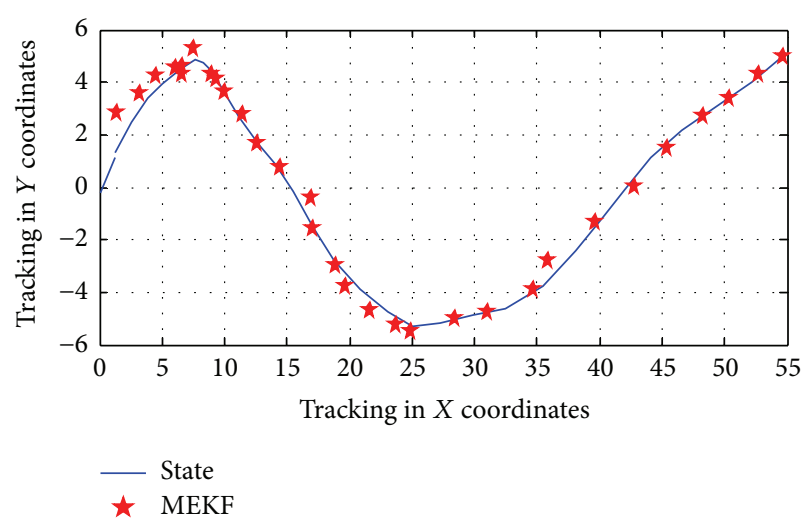

FIGURE 3: Tracking performance of the proposed MEKF.

TABLE 1: ADEs of the MEKF and the trilateration-based method for three cases of multiplicative noise.

\begin{tabular}{lcc}
\hline Multiplicative noise & \multicolumn{2}{c}{ Method } \\
\hline$M=\operatorname{diag}\{0.95,0.98,0.95\}$ & 0.1617 & Trilateration \\
$M=\operatorname{diag}\{0.9,0.8,0.9\}$ & 0.3673 & 0.4134 \\
$M=\operatorname{diag}\{0.8,0.7,0.7\}$ & 0.4151 & 0.6751 \\
\hline
\end{tabular}

The average distance error (ADE) is introduced to compare the tracking performances, which is defined as

$$
\mathrm{ADE}=\frac{\sum \sqrt{\left(x_{k}-\widehat{x}_{k \mid k}\right)^{2}+\left(y_{k}-\widehat{y}_{k \mid k}\right)^{2}}}{\text { Number of Estimations }} .
$$

Two kinds of noise parameters affecting the tracking performances are discussed: the multiplicative noise and the measurement noise.

5.2.1. Impact of Multiplicative Noise. Table 1 shows the ADEs of the MEKF and the trilateration-based method suffering three different types of multiplicative noise. The corresponding distance error comparisons are shown in Figure 4. As shown in Figure 4, compared to the trilateration-based method, the MEKF proposed performs better for all three cases.

It is evident that, with the increase of the effective packet arrival rate (corresponding to the increase of the positive definite matrix $M$ in Table 1), the estimation error becomes smaller and smaller for both methods. This is to be expected, since more measurements may provide more valuable information for updating the estimation. Moreover, it is worth pointing out that if $M$ decreases, the performance of the trilateration-based method will degrade considerably, while the tracking accuracy of our filter will be affected slightly. The reasons are as follows. The trilateration-based method requires at least three effective measurements for each location point. Due to the sparse anchor support and the unreliable communication link, the target cannot always be localized during its movement. The trace estimated by connecting localized positions with three measurements fails 


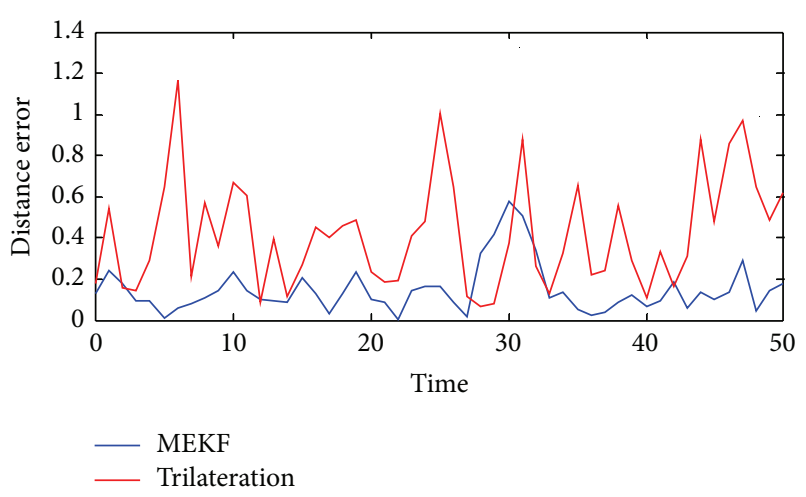

(a) Comparison with $M=\operatorname{diag}\{0.95,0.98,0.95\}$

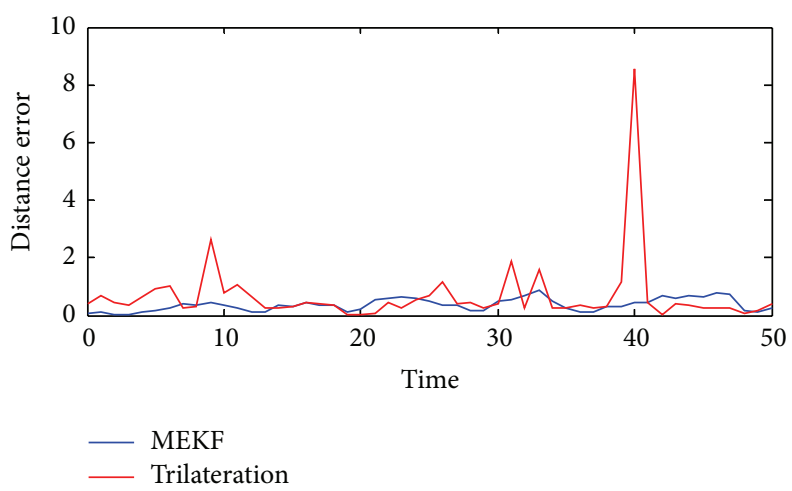

(b) Comparison with $M=\operatorname{diag}\{0.9,0.8,0.9\}$

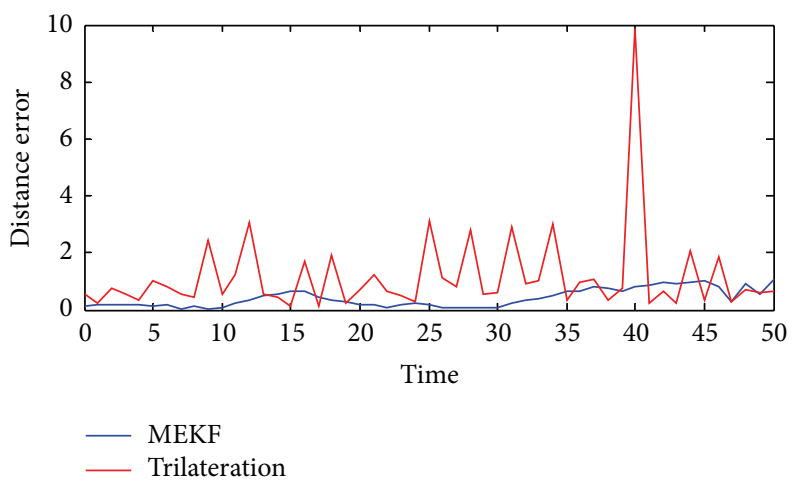

(c) Comparison with $M=\operatorname{diag}\{0.8,0.7,0.7\}$

FIGURE 4: Comparison curves under three different types of multiplicative noise.

to provide the high tracking accuracy. However, our algorithm given in Theorem 7 can propagate the state estimation of the previous time step and exploit information regarding the arrival partial observations sufficiently. As a result, it has a better performance under insufficient and intermittent observations.

5.2.2. Impact of Measurement Noise. The measurement noise would reduce the accuracy of the measurement signals and consequently degrade the tracking performance. In this subsection, we simulate the distance errors under different measurement noise. Figure 5 shows all of these cases, comparing the distance errors of our filter with those of the trilateration-based method. The detailed account of the corresponding ADEs is given in Table 2. As the covariance of the measurement noise increases, we can see the ADEs for both schemes increase.

It is easy to see that the ADE of our filter is smaller than that of the trilateration-based method, especially for the case of larger measurement noise covariance. This is not surprising, since the trilateration only depends on the distances between the target location and the locations of anchors (i.e., the measurement equation) to track the moving target, while our method exploits additional information from the state equation. As a consequence, our scheme is more suitable for the scenario assuming the state equation is available. It is also worth noting that there are some
TABLE 2: ADEs of the MEKF and the trilateration-based method for three cases of measurement noise.

\begin{tabular}{lcc}
\hline Measurement noise & \multicolumn{2}{c}{ Method } \\
& MEKF & Trilateration \\
\hline$R_{k}=\operatorname{diag}\{0.0016,0.0013,0.0015\}$ & 0.0878 & 0.1823 \\
$R_{k}=\operatorname{diag}\{0.012,0.010,0.011\}$ & 0.1382 & 0.2547 \\
$R_{k}=\operatorname{diag}\{0.043,0.039,0.041\}$ & 0.2491 & 0.4692 \\
\hline
\end{tabular}

sharp protrusions on the trilateration curves as shown in Figures 4 and 5, which indicate considerable estimation errors. However, our method gives smoother curves for all cases.

\section{Conclusions}

This paper considers the tracking problem in the context of distributed sensor networks. The anchors collect observation data and send them to the corresponding central filtering units. The observations may be insufficient or intermittent or both due to environment constraints and unreliable links. We propose the modified extended Kalman filtering algorithm suitable for the above cases. Moreover, the effect of various parameters on tracking performances is evaluated by numerical examples. Future works will consist in designing 


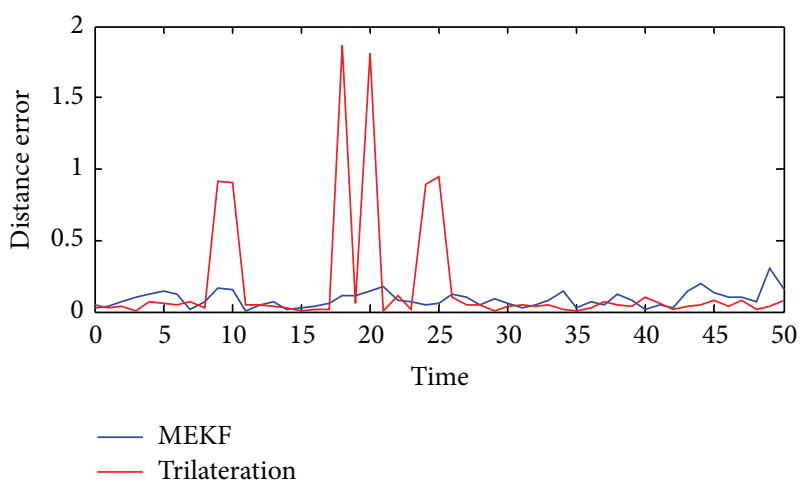

(a) Comparison with $R_{k}=\operatorname{diag}\{0.0016,0.0013,0.0015\}$

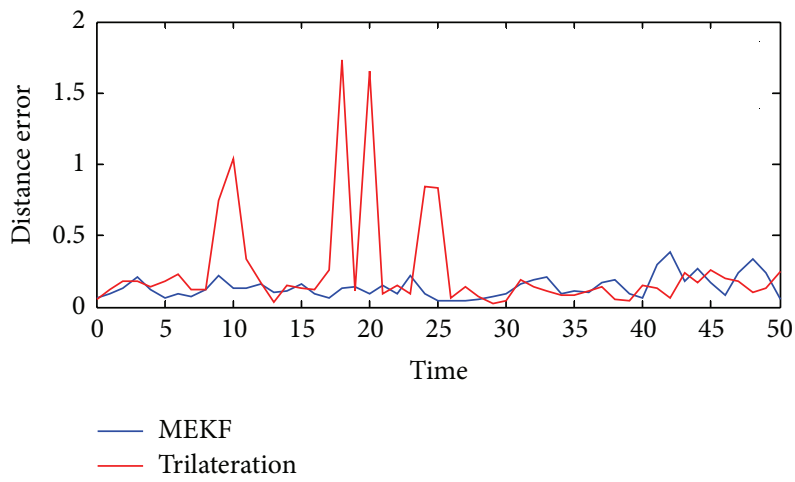

(b) Comparison with $R_{k}=\operatorname{diag}\{0.012,0.010,0.011\}$

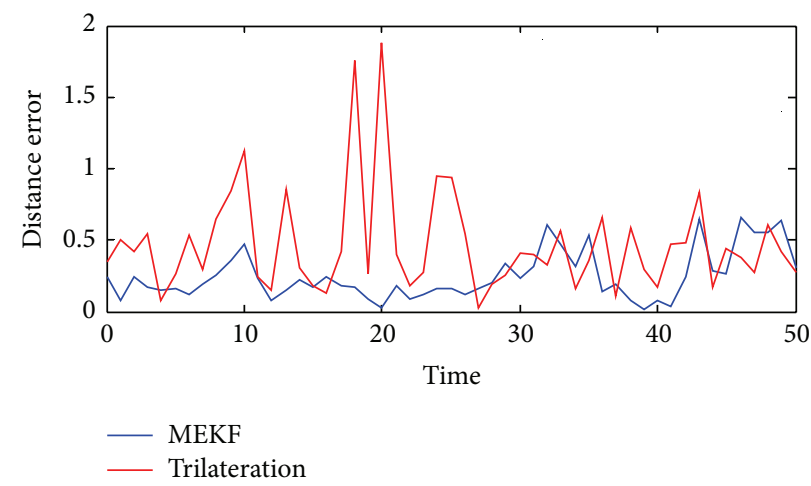

(c) Comparison with $R_{k}=\operatorname{diag}\{0.043,0.039,0.041\}$

FiguRE 5: Comparison curves under three different types of measurement noise.

and conducting experiments using the proposed MEKF algorithm.

\section{Conflict of Interests}

The authors declare that there is no conflict of interests regarding the publication of this paper.

\section{Acknowledgments}

This work was supported by the Fundamental Research Funds for the Central Universities under Grant 2012QNB18, the National Natural Science Foundation of China under Grant 61202478, and the Postdoctoral Science Foundation of China under Grant 2014M561727.

\section{References}

[1] F. M. Al-Turjman, H. S. Hassanein, and M. Ibnkahla, "Towards prolonged lifetime for deployed WSNs in outdoor environment monitoring," Ad Hoc Networks, vol. 24, pp. 172-185, 2015.

[2] M. Winkler, K. D. Tuchs, K. Hughes, and G. Barclay, "Theoretical and practical aspects of military wireless sensor networks," Journal of Telecommunications and Information Technology, vol. 2, pp. 37-45, 2008.

[3] A. A. Rezaee, M. H. Yaghmaee, and A. M. Rahmani, "Optimized congestion management protocol for healthcare wireless sensor networks," Wireless Personal Communications, vol. 75, no. 1, pp. 11-34, 2014.

[4] J. Jeong, S. Guo, Y. Gu, T. He, and D. H. C. Du, "Trajectorybased statistical forwarding for multihop infrastructure-tovehicle data delivery," IEEE Transactions on Mobile Computing, vol. 11, no. 10, pp. 1523-1537, 2012.

[5] X. Cheng, A. Thaeler, G. Xue, and D. Chen, "TPS: a time-based positioning scheme for outdoor wireless sensor networks," in Proceedings of the 23rd Conference of the IEEE Communications Society (INFOCOM '04), pp. 2685-2696, March 2004.

[6] J. Bai, P. Cheng, J. Chen, A. Guenard, and Y. Song, "Target tracking with limited sensing range in autonomous mobile sensor networks," in Proceedings of the 8th IEEE International Conference on Distributed Computing in Sensor Systems (DCOSS '12), pp. 329-334, May 2012.

[7] Y. Fu, Q. Ling, and Z. Tian, "Distributed sensor allocation for multi-target tracking in wireless sensor networks," IEEE Transactions on Aerospace and Electronic Systems, vol. 48, no. 4, pp. 3538-3553, 2012.

[8] G. Pillonetto, B. M. Bell, and S. Del Favero, "Distributed Kalman smoothing in static Bayesian networks," Automatica, vol. 49, no. 4, pp. 1001-1011, 2013.

[9] S. Movaghati and M. Ardakani, "Particle-based message passing algorithm for inference problems in wireless sensor networks," IEEE Sensors Journal, vol. 11, no. 3, pp. 745-754, 2011.

[10] L. Peng, X. Huang, and L. He, "Research on the target tracking algorithm for wireless sensor network based on improved particle filter," Journal of Convergence Information Technology, vol. 7, no. 11, pp. 11-19, 2012. 
[11] G. Lu, W. Zhao, J. Sun, S. Sun, and S. Mao, "A novel particle filter for target tracking in wireless sensor network," in Proceedings of the IET International Radar Conference, vol. 2013, no. 617 CP, April 2013.

[12] H.-J. Jang, J. W. Kim, and D.-H. Hwang, "Robust step detection method for pedestrian navigation systems," IEEE Electronics Letters, vol. 43, no. 14, pp. 749-751, 2007.

[13] B. Sinopoli, L. Schenato, M. Franceschetti, K. Poolla, M. I. Jordan, and S. S. Sastry, "Kalman filtering with intermittent observations," IEEE Transactions on Automatic Control, vol. 49, no. 9, pp. 1453-1464, 2004.

[14] X. Liu and A. Goldsmith, "Kalman filtering with partial observation losses," in Proceedings of the 43rd IEEE Conference on Decision and Control (CDC '04), pp. 4180-4186, December 2004.

[15] X. Lu and J. Chen, "Kalman filtering for multiple-delay wireless network systems with multiplicative noises," in Proceedings of the 10th IEEE International Conference on Control and Automation (ICCA' 13), pp. 259-263, June 2013.

[16] G. F. Welch and G. Bishop, An Introduction to the Kalman Filter, University of North Carolina, Chapel Hill, NC, USA, 1995.

[17] P. Chen, Z. Zhong, and T. He, "Bubble trace: mobile target tracking under insufficient anchor coverage," in Proceedings of the 31st International Conference on Distributed Computing Systems (ICDCS '11), pp. 770-779, July 2011.

[18] I. F. Akyildiz, W. Su, Y. Sankarasubramaniam, and E. Cayirci, "Wireless sensor networks: a survey," Computer Networks, vol. 38, no. 4, pp. 393-422, 2002.

[19] V. M. Moreno and A. Pigazo, Kalman Filter Recent Advances and Applications, Intech, Vienna, Austria, 2009.

[20] W. Y. Song and Y. Zhang, Kalman Filtering, Science Press, Beijing, China, 1991.

[21] D. Chu, Z. Zhang, and A. Zhao, "An iterative filter for a class of multi-channel nonlinear systems with multiplicative noise," in Proceedings of the 6th World Congress on Intelligent Control and Automation (WCICA '06), pp. 1529-1532, IEEE, Dalian, China, June 2006.

[22] S. Gao and P. Chen, "Suboptimal filtering of networked discretetime systems with random observation losses," Mathematical Problems in Engineering, vol. 2014, Article ID 151836, 8 pages, 2014. 


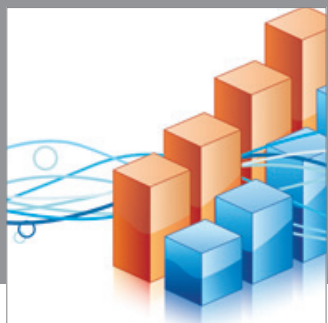

Advances in

Operations Research

mansans

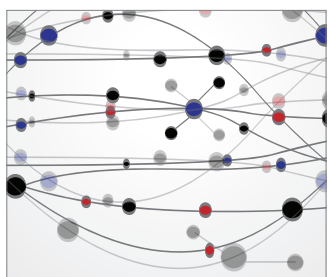

The Scientific World Journal
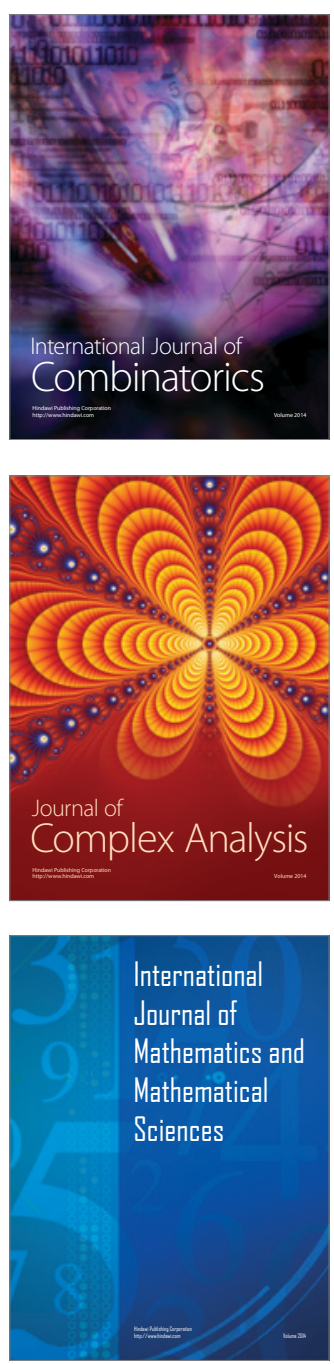
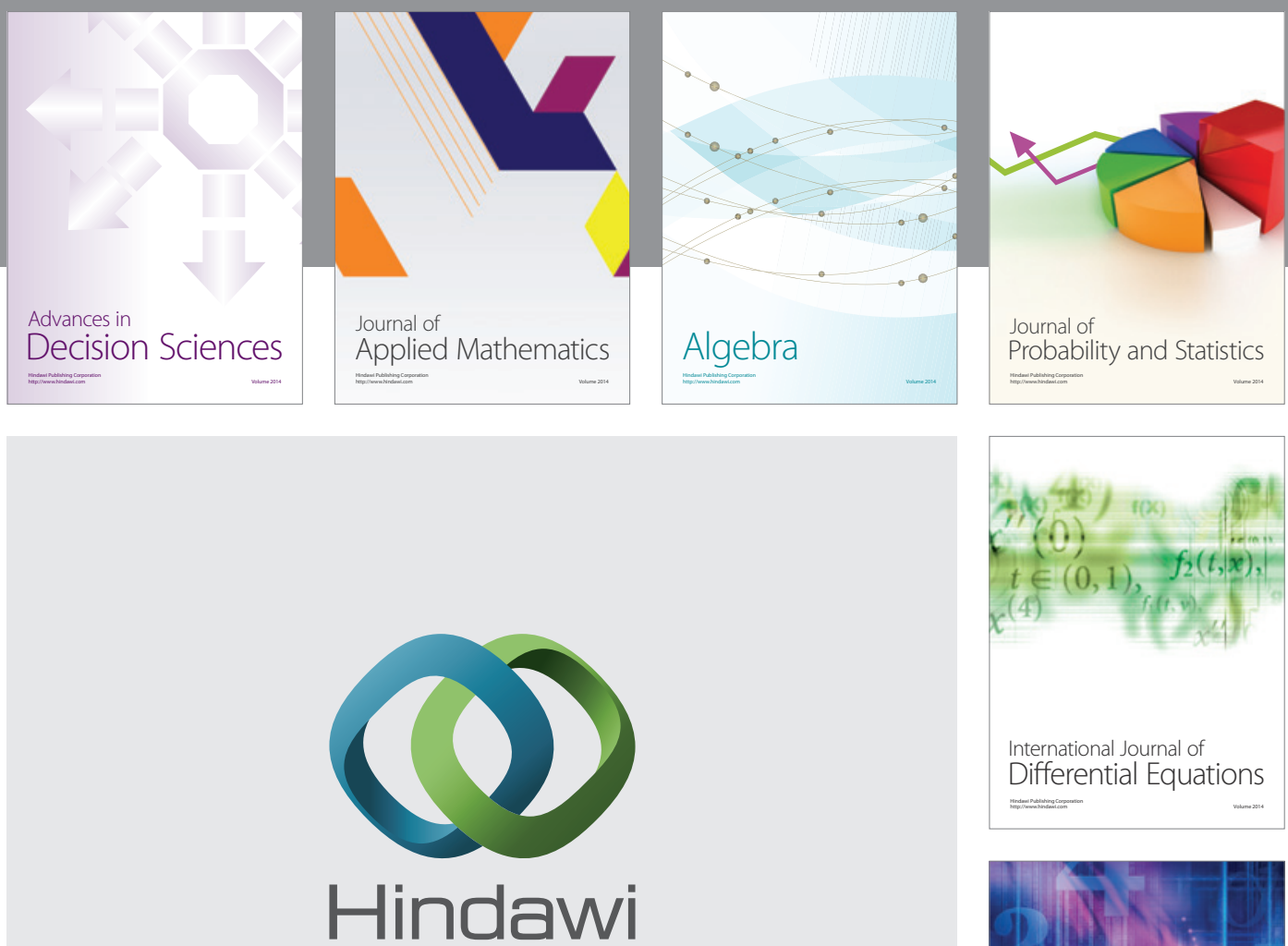

Submit your manuscripts at http://www.hindawi.com
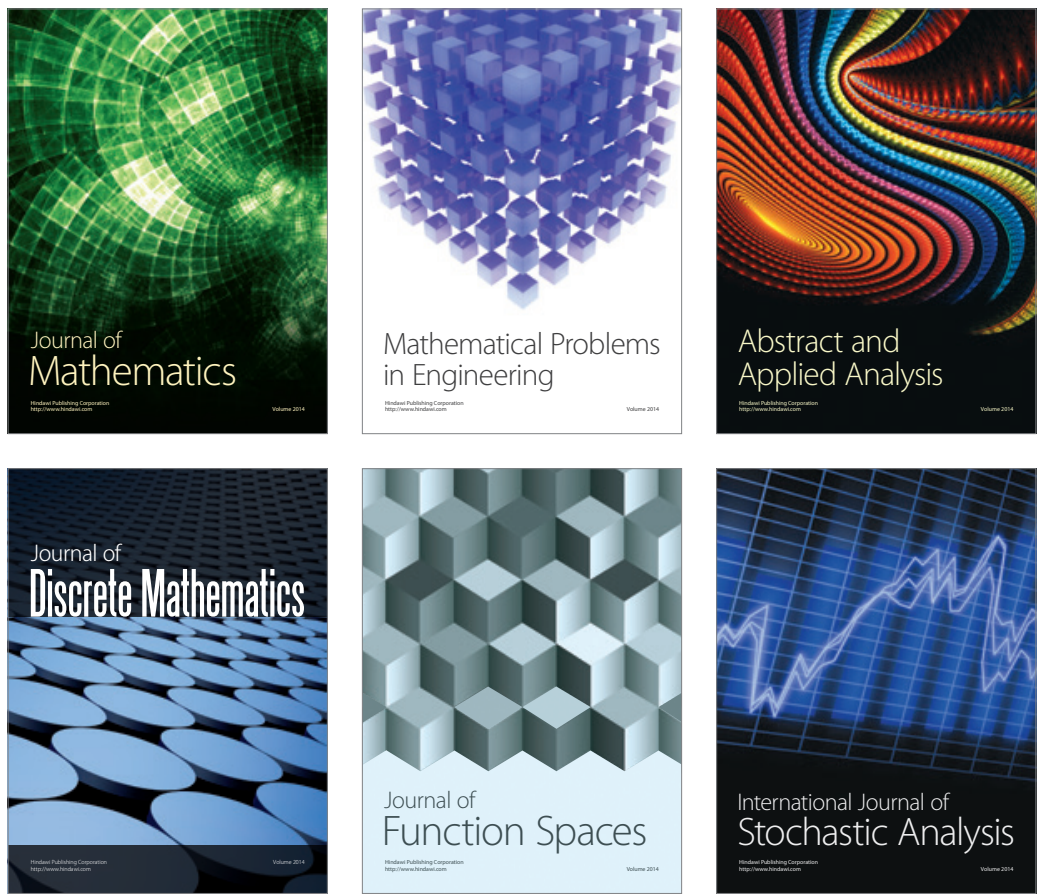

Journal of

Function Spaces

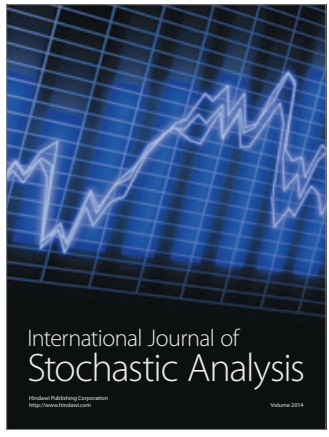

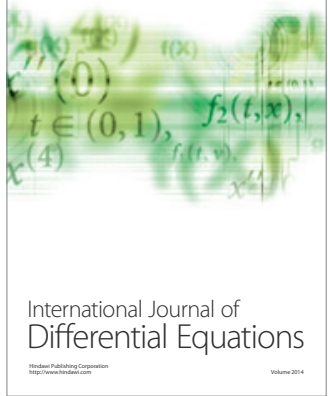
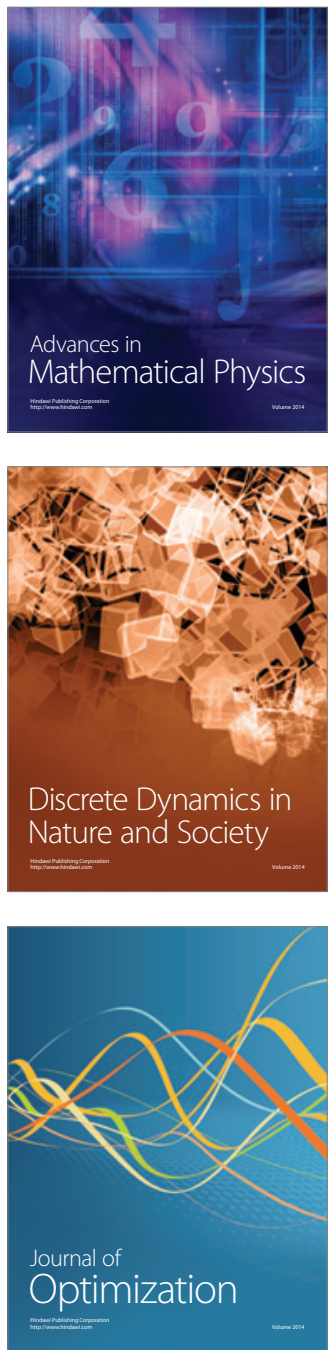